\title{
Reducing Communication Costs in Robust Peer-to-Peer Networks
}

\author{
Jared Saia* $\quad$ Maxwell Young*
}

\begin{abstract}
Several recent research results describe how to design Distributed Hash Tables (DHTs) that are robust to adversarial attack via Byzantine faults. Unfortunately, all of these results require a significant blowup in communication costs over standard DHTs. For example, to perform a lookup operation, all such robust DHTs of which we are aware require sending $O\left(\log ^{3} n\right)$ messages while standard DHTs require sending only $O(\log n)$, where $n$ is the number of nodes in the network. In this paper, we describe protocols to reduce the communication costs of all such robust DHTs. In particular, we give a protocol to reduce the number of messages sent to perform a lookup operation from $O\left(\log ^{3} n\right)$ to $O\left(\log ^{2} n\right)$ in expectation. Moreover, we also give a protocol for sending a large (i.e. containing $\Omega\left(\log ^{4} n\right)$ bits) message securely through a robust DHT that requires, in expectation, only a constant blowup in the total number of bits sent compared with performing the same operation in a standard DHT. This is an improvement over the $O\left(\log ^{2} n\right)$ bit blowup that is required to perform such an operation in all current robust DHTs. Both of our protocols are robust against an adaptive adversary.
\end{abstract}

Keywords: algorithms; distributed computing; fault-tolerance; peer-to-peer; adaptive adversary.

\section{Introduction and Related Work}

A distributed hash table (DHT) is a structured peer-to-peer (p2p) network which provides for scalable and distributed storage and lookup of data items (see e.g. [11, 9, 12]). Because peer-to-peer networks have little to no admission control, there has been significant effort in designing DHT's which are robust to Byzantine faults. When a peer suffers a Byzantine fault it is assumed to be controlled by an adversary who uses that peer to try to disrupt the network. We refer to the peers that suffer Byzantine faults as bad and the remaining peers as good.

Several recent results describe DHTs that are robust to adversarial attack via Byzantine faults [3, $5,7,4,1]$. The current state of the art in this area is a recent, striking result due to Awerbuch and Scheideler that describes a DHT that remains robust even if a number of join and leave events by Byzantine peers is polynomial in the size of the network $n$ [1]. All of these past results make use of quorums, which are sets of $\Theta(\log n)$ peers with the property that no more than a fixed constant fraction of the peers in a quorum have suffered Byzantine faults.

While each of these results creates and maintains quorums in a different manner, the results are similar in the way in which they use quorums to perform lookup operations. In particular, assume that a peer $p$ initiates the lookup operation and that there is a sequence of $l$ quorums, $Q_{1}, Q_{2}, \ldots, Q_{l}$, that the lookup request must pass through. The request is first forwarded from $p$ to $Q_{1}$. Next, for all $i$ from 1 to $l-1$, every peer in quorum $Q_{i}$ forwards the request to every peer in $Q_{i+1}$. Peers in $Q_{i+1}$ accept only those messages that are received from a majority of peers in $Q_{i}$. Finally, the requested data item is retrieved by quorum $Q_{l}$ and the data item is sent back in the same manner from $Q_{l}$ to $Q_{1}$ and then to $p$. Provided that a majority of peers in each quorum are not controlled by the adversary,

*Department of Computer Science, University of New Mexico, NM, USA; email: saia, young@cs.unm.edu. This research was partially supported by NSF grant CCR-0313160 and Sandia University Research Program grant No. 191445. 
this simple procedure ensures robust lookup operations. However, this technique is not bandwidth efficient. In particular, if $l=O(\log n)$, as is common, then the procedure requires $O\left(\log ^{3} n\right)$ messages. Moreover, if a data item containing $b$ bits is sent using this procedure, then the total number of bits sent is $O\left(b l \cdot \log ^{2} n\right)$.

Our Contributions: In this paper, we give two algorithms for efficient communication through quorums in a robust DHT. First, we give an algorithm to reduce the communication for lookups from $O\left(\log ^{3} n\right)$ messages to $O\left(\log ^{2} n\right)$ in expectation. Second, we give an algorithm for sending a large (i.e. containing $\Omega\left(\log ^{4} n\right)$ bits) message securely that requires, in expectation, only a constant blowup in the total number of bits sent compared with performing the same operation in a standard DHT. This is an improvement over the $O\left(\log ^{2} n\right)$ bit blowup that is required to perform such an operation in all previous quorum-based robust DHTs. Many first generation peer-to-peer systems sent large data items directly from the peer holding the data item to the peer requesting the item. However, increasingly in modern p2p systems such as BitTorrent, very large data items are being sent through the network to provide both 1) better download times for the receiver of the data item (due to increased bandwidth utilization); and 2) improved anonymity to the holder of the data item. To the best of our knowledge, our algorithm is the first with guaranteed robustness against a Byzantine adversary that will reduce bit blowup for modern p2p systems such as BitTorrent.

Each peer is assumed to have a unique ID (e.g. the IP address of the peer) and that peers in neighboring quorums know each others IDs. For simplicity, we will frequently use the same variable to refer both to the peer and to the ID of the peer. We will call peers controlled by the adversary bad and will call the remaining peers good. For both results, we assume that strictly less than a $1 / 4$ fraction of the peers in every quorum are bad. We also assume that each quorum is of size $C_{1} \ln n$ where $C_{1}$ is a positive constant ${ }^{1}$. A primary focus of all of the robust DHT results mentioned above is to describe protocols to maintain these types of invariants for the quorums. Thus, in this paper, we assume that these invariants are true and focus instead only on enabling efficient and secure communication.

Given these invariants, our protocols ensure correctness, with high probability ${ }^{2}$, over a polynomial number of peer join and leave events. Our first protocol is robust against a computationally unbounded adversary, while our second protocol is robust only against a polynomial-time bounded adversary. Our protocols are fully integrable with the p2p networks described in $[1,3,5,7,4]$. In addition, our protocols can be used to create more efficient protocols, not just looking up data items, but also for other p2p operations such as allowing a peer to join the network or maintaining link structure. A preliminary version of the results in this paper appeared in [4].

\section{Expected $\Theta\left(\log ^{2} n\right)$ Messages}

In this section, we show how to improve message passing between quorums so that only $\Theta(\log n)$ messages are sent in expectation from a quorum $L$ to a quorum $R$. To avoid the problem of the bad peers in $L$ flooding the good peers in $R$, there must be some systematic way to match up each good peer $r \in R$ to those peers in $L$ that should legitimately be sending to $r$. A naive approach for doing this would be to order peers in $L$ and $R$ by distance from the start of the quorum and then match up senders and receivers according to this ordering. Unfortunately, this approach fails since each peer may have a slightly different view of the set of peers in $L$ and $R$. In particular, if some peer $x$ does not know about even a single peer in $L$, then $x$ 's ordering of the peers in $L$ may be completely incorrect.

To remedy this problem, we make use of two functions to match up senders and receivers. We assume that the adversary has full knowledge of these functions before the protocol starts. Thus, the naive approach of matching up senders in $L$ and receivers in $R$ according to the output of a single function will fail. Instead, we match up senders and receivers via a more clever two-stage technique.

\footnotetext{
${ }^{1}$ We note that it is easy to generalize our results to the case that not all quorums are of the same size, but rather that each quorum is of size between $C_{1} \ln n$ and $C_{2} \ln n$ for fixed and known constants $C_{1}$ and $C_{2}$.

${ }^{2}$ We use the phrase with high probability throughout this paper to mean with probability $1-1 / n^{k}$ for any desired constant $k>0$.
} 


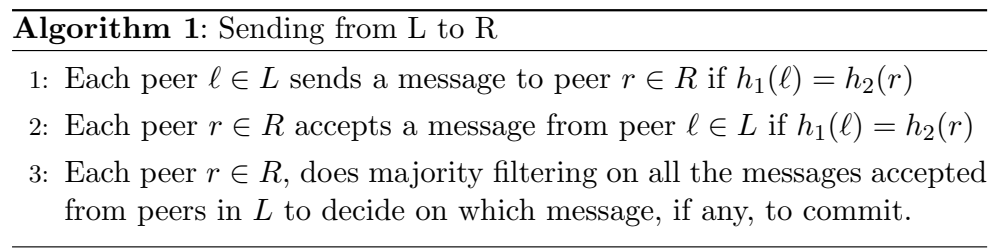

In particular, we assume all peers have functions $h_{1}$ and $h_{2}$ that map peers to the positive integers. The function $h_{1}$ maps a peer uniformly to an integer in the range 1 to $\ln n$, while $h_{2}$ maps a peer to $C_{2}$ integers selected uniformly and independently in the range 1 to $\ln n$, for some constant $C_{2}$ to be specified later. For ease of presentation, we assume the existence of functions $h_{1}$ and $h_{2}$ for the following analysis; we will show how such functions can be constructed at the end of Section 2.

We first say that each peer $\ell \in L$ is assigned to a bin between 1 and $\ln n$ whose number is given by $h_{1}(\ell)$. The bad peers in $L$ can choose the bin they are assigned to and thus the bad peers get to make their bin selections after seeing the bins chosen by all the good peers. However, we can still show that with high probability, at least a 6/10 fraction of the bins will be good in that they will contain a majority of good peers. Next, each good peer $r \in R$ is assigned to $C_{2}$ bins using $h_{2}(r)$. Finally, each peer $\ell \in L$ sends to every peer $r \in R$ that is assigned to the same bin as $\ell$. Then each peer $r \in R$ first does majority filtering over each bin it was assigned to in order to get a message value for that bin. Next, $r$ does majority filtering over the message values from each of the $C_{2}$ bins in order to decide on a single message value.

We then are able to show that no matter what set of $(4 / 10) \ln n$ of the bins are bad, that for $C_{2}$ chosen sufficiently large, almost all of the good peers in $R$ will be assigned to a majority of good bins and will thus decide on the correct message. To show this requires a somewhat subtle proof that makes use of the probabilistic method since there are many possibilities for which bins may be bad. Our algorithm for reducing message cost when sending from quorum $L$ to quorum $R$, is given in Algorithm 1 . The proof of correctness of our lookup procedure is given as Theorem 1.

Lemma 1. The expected number of messages sent by good peers in Algorithm 1 is $\Theta(\log n)$

Proof. There are $C_{1} \ln n$ peers in $R$ and each of them maps to $C_{2}$ bins. Thus a bin chosen uniformly at random will have $C_{1} C_{2}$ peers in $R$ that map to it. Each good peer in $L$ maps to a bin chosen uniformly at random so the expected number of messages sent by any good peer is $C_{1} C_{2}$. By linearity of expectation, the expected number of messages sent by all good peers is $C_{1} C_{2} \log n=\Theta(\ln n)$.

Recall that we say that a bin is good if among the peers in $L$ in that bin, a majority are good and have the correct message. The next lemma lower-bounds the number of good bins.

Lemma 2. If at least a 74/100 fraction of the peers in $L$ are good and have the correct message then for $C_{1}$ chosen sufficiently large, with high probability, at least a 6/10 fraction of the bins will be good.

Proof. We will say that a bin is full if it has at least $(7 / 10) C_{1}$ good peers in it (and any number of bad peers). We first show that for any $\epsilon>0$, we can choose $C_{1}$ such that, with high probability, all but $\epsilon \log n$ bins will be full. To see this, let $B^{\prime}$ be some set of $\epsilon \log n$ bins and let $N\left(B^{\prime}\right)$ be the number of good peers assigned to $B^{\prime}$. Further let $f=74 / 100$ be the fraction of good peers that have the correct message. Note that the expected number of good peers assigned to $B^{\prime}$ is exactly $f C_{1} \epsilon \ln n$. Further note that each good peer is assigned independently to $B^{\prime}$. Thus, by Chernoff bounds, we can say that for any $0 \leq \delta \leq 1$ :

$$
\operatorname{Pr}\left(N\left(B^{\prime}\right) \leq(1-\delta) f \epsilon C_{1} \epsilon \ln n\right) \leq e^{-(1 / 2) \delta^{2} f \epsilon C_{1} \ln n}
$$

We are particularly interested in the case where $\delta=4 / 74$ which ensures that $(1-\delta) f=7 / 10$. Let $\xi$ be the event that any set of $\epsilon \log n$ bins have less than $(1-\delta) f C_{1} \epsilon \ln n$ good peers in them. Then we can 
say that:

$$
\begin{aligned}
\operatorname{Pr}(\xi) & \leq\left(\begin{array}{c}
\ln n \\
\epsilon \ln n
\end{array}\right) e^{-(1 / 2) \delta^{2} f \epsilon C_{1} \ln n} \\
& \leq e^{\left(1-(1 / 2) \delta^{2} f \epsilon C_{1}\right) \ln n}
\end{aligned}
$$

For any $k>0$, any $\delta>0$, any $0<\epsilon<1$ and $C_{1}$ chosen greater than $\frac{2(1+k)}{f \epsilon \delta^{2}}$ this last probability will be no more than $n^{-k}$. Choosing, $\epsilon=1 / 100$ and $\delta=4 / 74$, we can say that w.h.p., no more than a $1 / 100$ fraction of the bins have less than $(7 / 10) C_{1}$ good peers in them.

We still must show that the adversary cannot place the bad peers in such a way as to take over too many bins. To make a full bin bad, the adversary must place at least $(7 / 10) C_{1}$ bad peers in the bin. The adversary has $(1 / 4) C_{1} \ln n$ bad peers to place so it can take over no more than $\frac{(1 / 4) C_{1} \ln n}{(7 / 10) C_{1}}=(5 / 14) C_{1} \ln n$ of the full bins. Even assuming that the adversary also takes over all of the bins that are not full, the number of bad bins will be no more than $(5 / 14+1 / 100) C_{1} \ln n<(4 / 10) C_{1} \ln n$. Thus, the fraction of good bins will be at least $6 / 10$.

Lemma 3. If at least a 6/10 fraction of the bins are good then for $C_{2}$ chosen sufficiently large, with high probability, at least $(74 / 100) C_{1} \ln n$ peers in $R$ will be good and will commit to the correct message.

Proof. We must show that the number of good peers in $R$ that do not map to a majority of good bins is no more than $(1 / 100) C_{1} \ln n$. Consider some set $R^{\prime}$ of good peers in $R$ where $\left|R^{\prime}\right|=(1 / 100) C_{1} \ln n$. Let $N\left(R^{\prime}\right)$ be the number of good bins that peers in $R^{\prime}$ map to. Each peer in $R^{\prime}$ maps to $C_{2}$ bins so $E\left(N\left(R^{\prime}\right)\right) \geq C_{2}(6 / 10)(1 / 100) C_{1} \ln n$. By Chernoff bounds, we can say that for any $0 \leq \delta \leq 1$ :

$$
\operatorname{Pr}\left(N\left(R^{\prime}\right) \leq(1-\delta) E\left(N\left(R^{\prime}\right)\right)\right) \leq e^{-\delta^{2} C_{2}(3 / 1000) C_{1} \ln n}
$$

We are particularly interested in the case where $\delta=1 / 6$ which ensures that $(1-\delta)(6 / 10)=1 / 2$. Let $\xi$ be the event that any set of $(1 / 100) \log n \operatorname{good}$ peers in $R$ all do not map to a majority of good bins. Then we can say that:

$$
\begin{aligned}
\operatorname{Pr}(\xi) & \leq\left(\begin{array}{c}
C_{1} \ln n \\
(1 / 100) C_{1} \ln n
\end{array}\right) e^{-\delta^{2} C_{2}(3 / 1000) C_{1} \ln n} \\
& \leq e^{\left(1-\delta^{2} C_{2}(3 / 1000)\right) C_{1} \ln n}
\end{aligned}
$$

Choosing $C_{2}>\frac{1000}{3 \delta^{2}}$ ensures that the lemma holds with high probability.

The following corollary is immediate from Lemmas 2 and 3.

Corollary 1. For $C_{1}$ and $C_{2}$ chosen sufficiently large, with high probability, if at least a 74/100 fraction of the peers in $L$ are good and have the correct message then at the end of Algorithm 1, at least a 74/100 fraction of the peers in $R$ will be good and have the correct message.

Let LOOKUP denote a lookup protocol consisting of communication through a sequence of $O(\log n)$ quorums.

Theorem 1. For $C_{1}, C_{2}$ sufficiently large but depending only on $k$, the following is true with probability at least $1-1 / n^{k}$ :

- All calls to LOOKUP succeed.

- All calls to LOOKUP send $\Theta\left(\log ^{2} n\right)$ messages in expectation. 


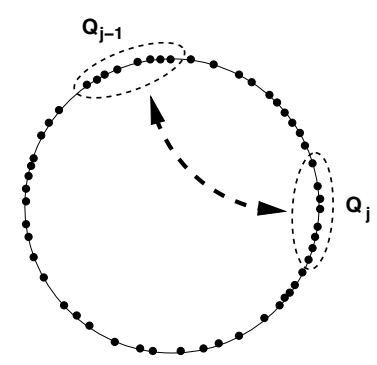

(A)

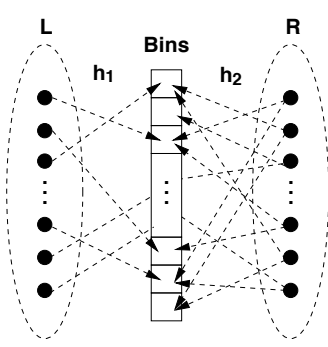

(B)

Figure 1: (A) Two quorums $Q_{j-1}$ and $Q_{j}$ on the unit circle in a DHT. The bidirectional dashed arrow implies that all peers in $Q_{j-1}$ know the IDs of all peers in $Q_{j}$ and vice-versa. (B) In the context of Algorithm 1, the quorums $L$ and $R$ are depicted. Each peer $\ell \in L$ is mapped to a single bin via $h_{1}$ and each peer $r \in R$ is mapped to $C_{2}$ bins via $h_{2}$ where in this simple depiction $C_{2}=2$.

Proof. Consider some arbitrary call to the lookup protocol described by the sequence: $p, Q_{1}, Q_{2}$, $Q_{3}, \ldots, Q_{l}$ where $p$ belongs to $Q_{1}$ and $m$ is the request sent. Here $p$ sends the message $m$ to quorum $Q_{1}, Q_{1}$ sends the request to $Q_{2}$ via Algorithm $1, Q_{2}$ sends the request to $Q_{3}$ via Algorithm 1 and so on. For a quorum $Q$, let $G(Q)$ be the set of good peers in $Q$ that commit to $m$ after majority filtering.

By a Union bound and repeated applications of Corollary 1, we can say that w.h.p., for any $Q_{i}, G\left(Q_{i}\right) \geq 74 / 100 C_{1} \ln n$. Thus $(74 / 100)\left|Q_{l}\right|$ good peers in $Q_{\ell}$ receive the request $m$ and fetch the appropriate data item, $d$. A similar argument from $Q_{\ell}, Q_{\ell-1}$ and all the way back to $Q_{1}$ shows that $p$ will receive the correct data item $d$ after majority filtering on messages received from $Q_{1}$. Finally, note that since $l=O(\log n)$, by Lemma 2, the expected number of messages sent is $\Theta\left(\log ^{2} n\right)$.

Finally, in order for Algorithm 1 to be correct, the following must be true. First, the good peers must be mapped by $h_{1}$ and $h_{2}$ to bins uniformly at random in the appropriate ranges. Second, the assignment of all peers to bins must be known unambiguously to all good peers in $L$ and $R$. In order to satisfy both conditions, the following protocol suffices. Upon joining the network at quorum $L$, a peer $p$ generates a bin number $b_{p}$ uniformly at random in $\{1, \ldots, \ln n\}$ using an internal random number generator. Bad peers may select their own bin number. Peer $p$ tells all peers in $L$ the value $b_{p}$. In order to prevent any ambiguity that might arise from a bad peer telling different peers in $L$ different values for $b_{p}$, all peers in $L$ come to consensus on the value received using a standard Byzantine agreement protocol. The peers in $R$ are subsequently informed via all-to-all communication between $R$ and $L$. In this way, peers in $L$ and $R$ know a peer's bin number unambiguously whenever it joins the network. The solution for constructing $h_{2}$ is essentially identical.

\section{Expected $\Theta(1)$ Bit Blowup}

In this section we assume that peer $p$ is trying to transmit a message $m$ along the path $p, Q_{1}, Q_{2}, Q_{3}$, $\ldots, Q_{l}$ where $Q_{i}, i=1, \ldots, l$ are quorums and $p$ belongs to $Q_{1}$. We assume that the adversary is polynomially bounded. Let $|m|$ be the number of bits in the message $m$. Peer $p$ first encodes $m$ into $\ln n$ pieces $e_{1}, e_{2}, \ldots, e_{\ln }$ with the following properties: 1) each piece has $O(|m| / \log n)$ bits and 2) $m$ can be reconstructed from any $1 / 16$-th fraction of the pieces. Any standard erasure code, such as tornado codes [6], can be used to create pieces with these two properties.

Peer $p$ next creates fingerprints $f_{1}, f_{2}, \ldots, f_{\ln n}$ of all these pieces using a 1-way hash function, $h_{f}$ known by all the peers. For all $i=1, \ldots, \ln n, f_{i}=h_{f}\left(e_{i}\right)$. Each of fingerprint has $\log ^{2} n$ bits. This ensures that the probability that a random string maps to a fixed fingerprint is $1 / n^{\ln n}$; therefore, the 
adversary requires superpolynomial time to find a string which maps to one of the fingerprints. We also make use of the function $h_{1}$ from the previous section that maps peers to integers in the range 1 to $\ln n$.

Peer $p$ sends all of the fingerprints to all of the peers in $Q_{1}$. Then for all $j=2, \ldots, l$, all peers in $Q_{j-1}$ send all of the fingerprints to all the peers in $Q_{j}$ and peers in $Q_{j}$ accept a fingerprint if and only if it was received from a majority of peers in $Q_{j-1}$. This guarantees that all peers in the quorums $Q_{1}, Q_{2}, \ldots, Q_{l}$ know all of the fingerprints. We will thus assume, in the protocol, described in this section, that a peer accepts a string $s$ as some piece $e_{j}$ if and only if $h_{f}(s)=f_{j}$.

The first step of our protocol is simple. Peer $p$ sends piece $e_{i}$ to peer $x \in Q_{1}$ iff $h_{1}(x)=i$. The general protocol where $Q_{j-1}$ sends to $Q_{j}$ for all $j=2, \ldots, l$ is given as Algorithm 2. This protocol makes use of a Byzantine agreement protocol and the protocol for coming to consensus on a random number. For the latter, we employ the scheme proposed in [1]. The basic idea of the remaining steps of the protocol i.e. sending from $Q_{j-1}$ to $Q_{j}$ is as follows. The peers in $Q_{j-1}$ agree on a randomly selected leader in $Q_{j}$ to whom they will send all their pieces. This leader, if good, will reconstruct the message $m$; reconstruct the pieces of $m$; and then send out the appropriate piece to each peer in $Q_{j}$. Each peer in $Q_{j}$ checks the piece they received against the fingerprint for that piece. The peers in $Q_{j}$ then run an agreement procedure to determine if enough of them received the correct piece. If so, the step ends. If not, this information is sent back to $Q_{j-1}$ and a new random leader is selected. For any quorum $Q$, we will now let $G(Q)=\left\{x \in Q \mid h_{1}(x)=i\right.$ and $x$ is good and has piece $\left.e_{i}\right\}$.

Lemma 4. For any fixed $k$, for $C_{1}$ sufficiently large but depending only on $k$, the following is true with probability at least $1-1 / n^{k}$. For all quorums $Q$, if $|G(Q)| \geq|Q| / 2$ and all peers in $Q$ send their pieces to some peer $x$, then $x$ will be able to reconstruct the message $m$.

Proof. We first fix a quorum $Q$ and calculate the probability that the statement of the lemma is not true. For any set of good peers, $X$, let $U(X)=\left\{i \mid h_{1}(x)=i\right.$, for some peer $\left.x \in X\right\}$. Let $Q^{\prime}$ be some fixed subset of good peers in $Q$ such that $\left|Q^{\prime}\right| \geq|Q| / 2$ and all peers in $Q^{\prime}$ have their correct pieces. Let $P^{\prime}$ be some fixed subset of the set of $\ln n$ pieces, such that $\left|P^{\prime}\right|>(15 / 16) \ln n$. Let $\xi\left(Q^{\prime}, P^{\prime}\right)$ be the probability that no peer in $Q^{\prime}$ has a piece in $P^{\prime}$. This is equivalent to a balls and bins problem where there are $\left|Q^{\prime}\right|$ balls and $\ln n$ bins and we are asking the probability that none of the $\left|Q^{\prime}\right|$ balls fall in a fixed set of $\left|P^{\prime}\right|$ of the bins. Thus:

$$
\operatorname{Pr}\left(\xi\left(Q^{\prime}, P^{\prime}\right)\right) \leq(1 / 16)^{\left|Q^{\prime}\right|} \leq(1 / 2)^{2|Q|}
$$

Now let $\xi(Q)$ be the event that for any subsets $Q^{\prime}$ and $P^{\prime}$, the event $\xi\left(Q^{\prime}, P^{\prime}\right)$ occurs. Then we have:

$$
\begin{aligned}
\operatorname{Pr}(\xi(Q)) & =\operatorname{Pr}\left(\bigcup_{Q^{\prime}, P^{\prime}} \xi\left(Q^{\prime}, P^{\prime}\right)\right) \leq \sum_{Q^{\prime}, P^{\prime}} \operatorname{Pr}\left(\xi\left(Q^{\prime}, P^{\prime}\right)\right)=\left(\begin{array}{c}
|Q| \\
|Q| / 2
\end{array}\right)\left(\begin{array}{c}
\ln n \\
(15 / 16) \ln n
\end{array}\right)(1 / 2)^{2|Q|} \\
& \leq 2^{|Q|} 2^{\ln n}\left((1 / 2)^{2|Q|}\right) \leq(1 / 2)^{|Q|-\ln n} \leq 1 / n^{C_{1}-1} .
\end{aligned}
$$

Where the last inequality follows since $|Q| \geq C_{1} \ln n$. A simple union bound over all $n$ of the swarms gives that the probability that the statement in the lemma fails for any quorum is no more than $1 / n^{C_{1}-2}$. Choosing $C_{1}$ sufficiently large makes this probability no more than $1 / n^{k}$ for any $k$.

We will refer to one iteration of the loop in Algorithm 2 as a round.

Lemma 5. For any fixed $k$, for $C_{1}$ sufficiently large but depending only on $k$, the following is true with probability at least $1-1 / n^{k}$ for all pairs of quorums, $Q_{j-1}$ and $Q_{j}$. If $\left|G\left(Q_{j-1}\right)\right| \geq(1 / 2)\left|Q_{j-1}\right|$ before Algorithm 2 starts, then $\left|G\left(Q_{j-1}\right)\right| \geq(1 / 2)\left|Q_{j}\right|$ after termination. Further, if all peers in $Q_{j-1}$ know all of the fingerprints before Algorithm 2 starts, then all peers in $Q_{j}$ will know all the fingerprints after termination. Algorithm 2 will:

- Terminate in $O(1)$ rounds in expectation;

- Require good peers to send $O\left(\log ^{3} n\right)$ messages in expectation; 


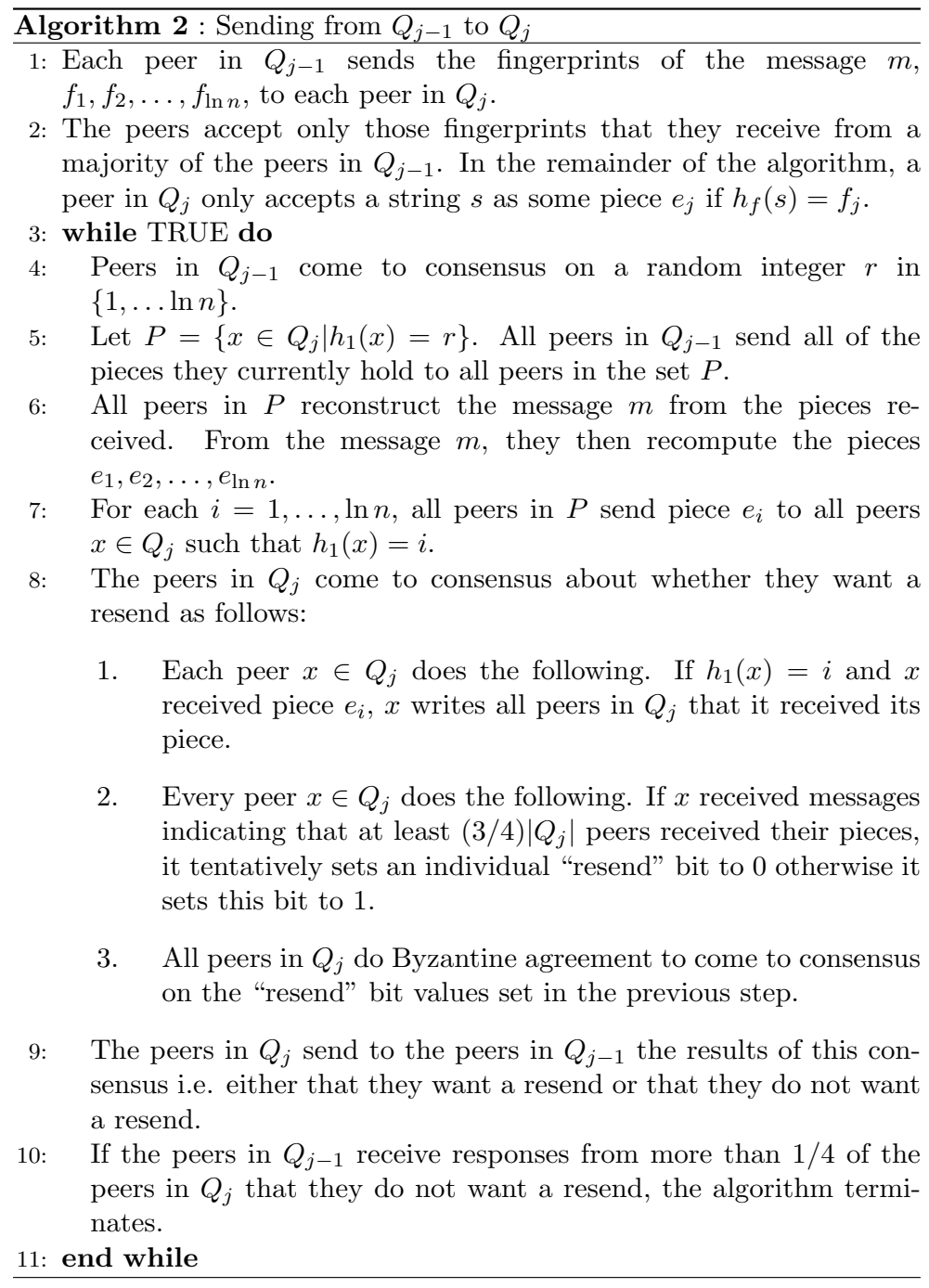

- Require good peers to send $O\left(|m|+\log ^{4} n\right)$ bits to be sent in expectation.

Proof. Since quorum $Q_{j-1}$ is good and the peers in $Q_{j}$ do majority filtering in Step 2, we know that if all peers in $Q_{j-1}$ know all of the fingerprints before Algorithm 2 starts, then all peers in $Q_{j}$ will know all the fingerprints after termination.

If $\left|G\left(Q_{j-1}\right)\right| \geq(1 / 2)\left|Q_{j-1}\right|$, then by Lemma 4, all peers in the set $P$ which are sent the message pieces in Step 5 will be able to reconstruct the message $m$. Since $3 / 4$ of the peers in $Q_{j}$ are good, the probability that no peer in $P$ is good is no more than $(1-1 / \ln n)^{(3 / 4) C_{1} \ln n} \leq e^{-(3 / 4) C_{1}}$. Thus with constant probability, some peer in the set $P$ is good. If this is the case, then all peers in $Q_{j}$ will receive their correct piece of the message. This implies that the algorithm will terminate in that round with $\left|G\left(Q_{j}\right)\right| \geq 1 / 2\left|Q_{j}\right|$. This implies that Algorithm 2 will terminate in an expected constant number of rounds.

We next establish correctness. Consider the situation where no peer in $P$ is good. There are then two possible cases. First is the case that less than $(1 / 2)\left|Q_{j}\right|$ peers in $Q_{j}$ are sent their pieces in Step 5. In this case, no peer in Step 8.2 will receive at least $(3 / 4)\left|Q_{j}\right|$ messages saying that pieces were received. Thus all good peers will set their resend bits to 1 in this step and so the consensus will be to request a resend. This implies that the algorithm will continue for another round. The second case is that faulty peers in $P$ send pieces to at least $(1 / 2)\left|Q_{j}\right|$ peers in $Q_{j}$. If this is the case, then it's safe for 
the algorithm to terminate.

We now compute the resource costs. Previous to the first round, the fingerprints are sent which requires $O\left(\log ^{2} n\right)$ messages, and $O\left(\log ^{4} n\right)$ bits. The expected size of the set $P$ is $O(1)$. We employ techniques from secure multiparty computation in order to have quorums select a random value in $[0,1)$. There are several results showing how to achieve secure multiparty computation in an asynchronous network provided that the fraction of Byzantine players is strictly less than $1 / 4$ (see $[2,10,8]$ ). Srinathan and Rangan [10] give the most resource efficient secure multiparty computation protocol of which we are aware for this problem. In the case where there are $\Theta(\log n)$ peers, strictly less than $1 / 4$ of which are faulty, we can compute a random number using $\Theta\left(\log ^{3} n\right)$ messages, with $\Theta(\log n)$ latency using their protocol. This protocol can be used to complete Step 3 in Algorithm 2. Therefore, in each round of the algorithm, the total number of messages sent is $O\left(\log ^{3} n\right)$ and the expected total number of bits sent is $O\left(|m|+\log ^{4} n\right)$. The expected resource costs of the entire algorithm then follow directly from the fact that there are $O(1)$ rounds in expectation.

Lemma 6. For any fixed $k$, for $C_{1}$ sufficiently large but depending only on $k$, the following is true with probability at least $1-1 / n^{k}$ if we run Algorithm 2:

- All calls to LOOKUP succeed.

- All calls to LOOKup have latency $O(\log n)$ in expectation.

- All calls to LOOKuP require $O\left(\log ^{4} n\right)$ messages to be sent in expectation.

- All calls to LoOKup require $O\left(|m| \log n+\log ^{5} n\right)$ bits to be sent in expectation.

Proof. Consider a message $m$ which is sent along a path described by the sequence: $p, Q_{1}, Q_{2}, Q_{3}, \ldots, Q_{l}, q$, where $p$ belongs to $Q_{1}$ and $q$ belongs to $Q_{l}$. Here peer $p$ sends all pieces of $m$ and fingerprints of these pieces to $Q_{1}=Q(p), Q_{2}$ sends the pieces and fingerprints to $Q_{2}$ via Algorithm 2, $Q_{2}$ sends the pieces and fingerprints to $Q_{3}$ via Algorithm 2 and so on until finally all peers in quorum $Q_{l}$ sends all their pieces and fingerprints to peer $q$. Since, $\left|G\left(Q_{1}\right)\right| \geq 1 / 2\left|Q_{1}\right|$, Lemma 5 and induction give that $\left|G\left(Q_{l}\right)\right| \geq 1 / 2\left|Q_{j}\right|$ and that all peers in $Q_{l}$ have all the fingerprints of these pieces. This implies that when peers in $Q_{l}$ send their pieces and fingerprints to $q, q$ will have enough information to reconstruct the message $m$. The latency, message complexity, and bit complexity follow immediately from Lemma 5 and the fact that $l \in O(\log n)$.

\section{Conclusion}

We have presented new algorithms for reducing communication costs in peer-to-peer networks that are robust against a dynamic adversary. Our algorithms are compatible with any type of $\mathrm{p} 2 \mathrm{p}$ network that maintains quorums with strictly less than a $1 / 4$ fraction of Byzantine nodes in each quorum. Many problems remain open including the following. We would like to prove lower-bounds on communication costs and the adversarial power that we can tolerate. Are $O\left(\log ^{2} n\right)$ messages necessary to perform a robust lookup? Can we tolerate more than a $1 / 4$ fraction of bad peers in each quorum? Can we reduce the bit blowup to $O(1)$ even against a computationally unbounded adversary? Finally, we would like to demonstrate that these algorithms are practical through simulation and possible use in a deployed system.

Acknowledgements: We gratefully thank Amos Fiat for his help with this paper.

\section{References}

[1] Baruch Awerbuch and Christian Scheideler. Towards a Scalable and Robust DHT. In Proceedings of 18th ACM Symposium on Parallelism in Algorithms and Architectures (SPAA), pages 318-327, 2006. 
[2] Michael Ben-Or,Ran Canetti and Oded Goldreich. Asynchronous Secure Computation. In Proceedings of the Twenty-Fifth ACM Symposium on the Theory of Computing (STOC), pages 52-61, 1993.

[3] Amos Fiat and Jared Saia. Censorship Resistant Peer-to-Peer Content Addressable Networks. In Proceedings of the Thirteenth ACM Symposium on Discrete Algorithms (SODA), pages 94-103, 2002.

[4] Amos Fiat, Jared Saia and Maxwell Young. Making Chord Robust to Byzantine Attacks. In Proceedings of the $13^{\text {th }}$ Annual European Symposium on Algorithms (ESA), pages 803-814, 2005.

[5] Kristen Hildrum and John Kubiatowicz. Asymptotically Efficient Approaches to Fault-Tolerance in Peer-to-peer Networks. In Proceedings of the 17th International Symposium on Distributed Computing, pages 321-336, 2004.

[6] Michael G. Luby, Michael Mitzenmacher, M. Amin Shokrollahi, Daniel A. Spielman and Volker Stemann. Practical Loss-Resilient Codes. In Proceedings of the Twenty-Ninth Annual ACM Symposium on Theory of Computing, pages 150-159, 1997.

[7] Moni Naor and Udi Wieder. A Simple Fault Tolerant Distributed Hash Table. In Proceedings of the Second International Workshop on Peer-to-Peer Systems (IPTPS), pages 88-97, 2003.

[8] B. Prabhu, K. Srinathan and C. Pandu Rangan. Asynchronous Unconditionally Secure Computation: An Efficiency Improvement. In INDOCRYPT 2002, Lecture Notes in Computer Science, volume 2551, pages 93-107. Springer-Verlag, 2002.

[9] Sylvia Ratnasamy, Paul Francis, Mark Handley, Richard Karp and Scott Shenker. A Scalable Content-Addressable Network. In Proceedings of the 2001 Conference on Applications, Technologies, Architectures, and Protocols for Computer Communications, pages 161-172, 2001.

[10] K. Srinathan and C. Pandu Rangan. Efficient Asynchronous Secure Multiparty Distributed Computation. In INDOCRYPT 2000, Lecture Notes in Computer Science, volume 1977, pages 117-129. Springer-Verlag, 2000.

[11] Ion Stoica, Robert Morris, David Karger, M. Frans Kaashoek and Hari Balakrishnan. Chord: A Scalable Peer-to-peer Lookup Service for Internet Applications. In Proceedings of the 2001 ACM SIGCOMM Conference, pages 149-160, 2001.

[12] Ben Y. Zhao, John Kubiatowicz and Anthony D. Joseph. Tapestry: An Infrastructure for FaultResilient Wide-Area Location and Routing. Technical Report UCB//CSD-01-1141, University of California at Berkeley, April 2001. 